\title{
Great Matrix Equations
}

\author{
Jumaboyevich $\mathrm{KN}^{*}$, Yuldashevich $\mathrm{AQ}$ and Ugli IOB
}

Department of Mathematics, Faculty of Physics and Mathematics, Urgench State University, Urgench, Uzbekistan

\begin{abstract}
This paper is devoted to some identities of matrix and determinants. In this article, matrices inequalities are widely used. Matrices are non-optimistic. The matrix's determinants have been proven. In addition to the left module and the right module descriptions in this article. In general, this article is devoted to matrix approximation.
\end{abstract}

Keywords: Matrix; Equation; Inequality; Number; Algebraic operations; Matrix multiplication; Triangular matrices

\section{Introduction}

Matrix is a mathematical object, written in the form of a rectangular table of ring or field elements (for example, integers, real numbers or complex numbers), which is a collection of rows and columns, at the intersection of which are its elements. The number of rows and columns determines the size of the matrix. Although historically considered, for example, triangular matrices [1], now they speak exclusively about matrices of rectangular shape, since they are the most convenient and common. Matrices are widely used in mathematics for the compact recording of systems of linear algebraic or differential equations. In this case, the number of rows of the matrix corresponds to the number of equations [2-4], and the number of columns to the number of unknowns. As a result, the solution of systems of linear equations reduces to operations on matrices. For the matrix the following algebraic operations are defined: Addition of matrices having the same size, multiplication of matrices of a suitable size (a matrix having display style $\mathrm{n}$ ) $\mathrm{n}$ columns can be multiplied from the right by a matrix having (display style in rows) [5-8], including multiplication by the matrix of the vector (according to the usual rule of matrix multiplication, the vector is in this sense a particular case of the matrix), multiplication of the matrix by an element of the base ring or field (that is, a scalar). Relative to the addition of a matrix form an Abelian group [9-12]; if we also consider multiplication by a scalar, then the matrices form a module over the corresponding ring (the vector space over the field). The set of square matrices is closed with respect to matrix multiplication; therefore square matrices of the same size form an associative ring with a unit with respect to matrix addition and matrix multiplication [13-16].

It is proved that to every linear operator acting in an $\mathrm{n}$-dimensional linear space one can associate a unique square matrix of order $\mathrm{n}$; and conversely - for each square matrix of order $n$, a single linear operator acting in this space can be associated. The properties of the matrix correspond to the properties of the linear operator. In particular, the eigenvalues of the matrix are the eigenvalues of the operator corresponding to the corresponding eigenvectors [17].

The same can be said about the representation of bilinear (quadratic) forms by matrices [2].

In mathematics, many different types and types of matrices are considered. These are, for example, a single, symmetric, skewsymmetric, upper triangular (lower triangular), etc. matrix [18].

Of particular importance in the theory of matrices are all possible normal forms, that is, the canonical form, to which the matrix can be reduced by the replacement of coordinates. The most important (theoretically) and well-developed is the theory of Jordan normal forms. In practice, however, such normal forms are used that have additional properties, for example, resistance [19].

The concept of matrix algebra is important in modeling the systems. Planning issues, gross product, total labor costs, pricing, and other issues, and the use of computers in them, will lead to the matrix algebra. Production planning should be based on a well-organized system of information, in the formulation of existing links between material production and others. This systematic information system is presented in the form of specific tables. Thus, the matrix's interpretation is quite extensive. In today's article, we create the theoretical theorems about the approximation of the matrix characteristics and matrices. Using proven theorems, we create mathematical comprehension patterns with each other [20]. We know that matrices and determinants are one of the main parts of algebra numbers.

$\mathrm{C}[\mathrm{mxm}]$ we define a set of matrices that are all elements of this form m-matrix order.

\section{Module of Matrix}

\section{Theorem 1.1}

$\forall A_{i} \in C[m, m]$ matrices and $a_{j} \in R j=\overline{1, n}$ For real numbers, the following matrix is valid.

$$
\sum_{k=1}^{n}\left|a_{k} \sum_{j=1}^{n} a_{j} A_{j}-A_{k} \sum_{j=1}^{n} a_{j}^{2}\right|^{2}=\sum_{j=1}^{n} a_{j}^{2}\left|\sum_{k=1}^{n} a_{k}^{2} \sum_{k=1}^{n} A_{k}\right|^{2}-\left|\sum_{k=1}^{n} a_{k} A_{k}\right|^{2}
$$

In this theorem: $|\circ|$-left or right module the left and right modules of the matrix are introduced as follows

$$
\begin{aligned}
& |A|_{R}=\sqrt{A A^{*}} \text { - right module } \\
& |A|_{L}=\sqrt{A^{*} A} \text { - left module. }
\end{aligned}
$$

The result: $\forall A_{i} \in C[m, m]$ matrices and $\forall a_{i} \in \square,(i=\overline{1 . n})$ for the real numbers

${ }^{*}$ Corresponding author: Jumaboyevich KN, Department of Mathematics, Faculty of Physics and Mathematics, Urgench State University, Urgench, Uzbekistan, Tel: +351932183080; E-mail: khujatovphd@mail.ru

Received December 27, 2017; Accepted January 24, 2018; Published January 30, 2018

Citation: Jumaboyevich KN, Yuldashevich AQ, Ugli IOB (2018) Great Matrix Equations. J Appl Computat Math 7: 384. doi: 10.4172/2168-9679.1000384

Copyright: () 2018 Jumaboyevich KN, et al. This is an open-access article distributed under the terms of the Creative Commons Attribution License, which permits unrestricted use, distribution, and reproduction in any medium, provided the original author and source are credited. 


$$
\sum_{i=1}^{n} a_{i}^{2} \sum_{i=1}^{n}\left|A_{i}\right|^{2}-\left|\sum_{i=1}^{n} a_{i} A_{i}\right|^{2} \geq 0
$$

Inequality is reasonable. In this $\mathrm{A} \geq 0, A$ indicates that the matrix is positive.

Prove of the theorem 1:

We look at this view: $A=\sum_{j=1}^{n} a_{j} A_{j}, a=\sum_{j=1}^{n} a_{j}^{2}$

We know this equation

$$
\begin{aligned}
& |A|^{2}=A A^{*}+A A^{*}=A\left(\sum_{j=1}^{n} a_{j} A_{j}\right)^{*}+\left(\sum_{j=1}^{n} a_{j} A_{j}\right) A^{*}= \\
& =\sum_{j=1}^{n}\left(a_{j} A A_{j}^{*}+a_{j} A_{j} A^{*}\right)
\end{aligned}
$$

In that case

$$
\begin{aligned}
& \sum_{k=1}^{n}\left|a_{k} A-A_{k} a\right|^{2}=\sum_{k=1}^{n}\left(a_{k} A-A_{k} a\right)\left(a_{k} A^{*}-A_{k}^{*} a\right)= \\
& =\sum_{k=1}^{n}\left(a_{k}^{2} A A^{*}-a_{k} a A A_{k}^{*}-a a_{k} A_{k} A^{*}+a^{2} A_{k} A_{k}^{*}\right)= \\
& =a|A|^{2}-a \sum_{k=1}^{n}\left(a_{k} A A^{*}+a_{k} A_{k} A^{*}\right)+a^{2} \sum_{k=1}^{n}\left|A_{k}\right|^{2}= \\
& a|A|^{2}-2 a|A|^{2}-2 a|A|^{2}+a^{2} \sum_{k=1}^{n}\left|A_{k}\right|^{2}=a^{2} \sum_{k=1}^{n}\left|A_{k}\right|^{2}-a|A|^{2}= \\
& =a\left(a \sum_{k=1}^{n}\left|A_{k}\right|^{2}-|A|^{2}\right)=\sum_{j=1}^{n} a_{j}^{2}\left(\sum_{k=1}^{n} a_{k}^{2} \sum_{k=1}^{n}\left|A_{k}\right|^{2}-\left|\sum_{k=1}^{n} a_{k} A_{k}\right|^{2}\right)
\end{aligned}
$$

Theorem proved.

\section{Theorem 1.2}

$\forall A, B \in C[m \times m]$ For matrices, the following equation holds.

$\operatorname{det}(\mathrm{I}+\mathrm{AB})=\operatorname{det}(\mathrm{I}+\mathrm{BA})$ Prove of the theorem№2: Consider the following situation

\section{Condition 1}

A-matrix contradictory or $\operatorname{det} \mathrm{A} \neq 0$.

We write the following equations:

$\mathrm{I}+\mathrm{AB}=\mathrm{AA}^{-1}+\mathrm{AB}=\mathrm{A}\left(\mathrm{A}^{-1}+\mathrm{B}\right)$

$\mathrm{I}+\mathrm{BA}=\mathrm{A}^{-1} \mathrm{~A}+\mathrm{BA}=\left(\mathrm{A}^{-1}+\mathrm{B}\right) \mathrm{A}$

Now let's write them down in the form below $\operatorname{Det}(I+A B)=\operatorname{det} A(A$ $\left.{ }^{1}+\mathrm{B}\right)=\operatorname{det} \mathrm{Adet}\left(\mathrm{A}^{-1}+\mathrm{B}\right)$

$\operatorname{det}(\mathrm{I}+\mathrm{BA})=\operatorname{det}\left(\mathrm{A}^{-1}+\mathrm{B}\right) \mathrm{A}=\operatorname{det}\left(\mathrm{A}^{-1}+\mathrm{B}\right) \operatorname{det} \mathrm{A}$

So the following equation is appropriate.

$\operatorname{det}(\mathrm{I}+\mathrm{AB})=\operatorname{det}(\mathrm{I}+\mathrm{BA})$

\section{Condition 2}

$\operatorname{Det} \mathrm{A}=0$

We will study the m-ordered non-linear equations.

$\operatorname{det}\left(\mathrm{A}_{\lambda}\right)=0$
Where $A_{\lambda}=A-\lambda I$ I- unity matrix.

According to the fundamental theorem of algebra, this equation has the root. Let this equation be as follows

$$
\left\{\lambda_{1}, \lambda_{2}, \lambda_{3}, \lambda_{n}\right\}
$$

Then the following equation corresponds to the optional $\lambda$ $\forall \lambda \in C \backslash\left\{\lambda_{1}, \lambda_{2}, \lambda_{3}, \cdots \lambda_{n}\right\} \operatorname{det}\left(\mathrm{A}_{\lambda}\right) \neq 0$. It follows from the of

$\mathrm{A}_{\lambda}$ inverse.

\section{Condition 3}

$\operatorname{det}\left(I+A_{\lambda} B\right)=\operatorname{det}\left(I+B A_{\lambda}\right)$

equality is appropriate.

Using the determinant's continuous function, $\lambda \rightarrow 0$ and we will create the following:

$$
\begin{aligned}
& \lim _{\lambda \rightarrow 0} \operatorname{det}\left(I+A_{\lambda} B\right)=\operatorname{det} \lim _{\lambda \rightarrow 0}\left(I+A_{\lambda} B\right)=\operatorname{det}(I+A B) \\
& \lim _{\lambda \rightarrow 0} \operatorname{det}\left(I+B A_{\lambda}\right)=\operatorname{det} \lim _{\lambda \rightarrow 0}\left(I+B A_{\lambda}\right)=\operatorname{det}(I+B A)
\end{aligned}
$$

So the following equation is appropriate

$$
\operatorname{det}(I+A B)=\operatorname{det}(I+B A) \text {. }
$$

\section{Condition 4}

1. Equality has been proven for private by the method [3]. For example: for $\mathrm{A}=\mathrm{Z}, \mathrm{B}=\mathrm{Z}^{*}$

\section{Annex 1: Eqn. (5) is proved differently.}

Annex 2: For $\forall \mathrm{A}, \mathrm{B} \in \mathrm{C}[\mathrm{mXm}]$ equality $\mathrm{AB}=\mathrm{BA}$, in general it is not appropriate. We have demonstrated that this equality is appropriate, using several methods $\operatorname{det}(\mathrm{I}+\mathrm{AB})=\operatorname{det}(\mathrm{I}+\mathrm{BA})$.

\section{Conclusion}

In summary, it must be said that equality in the world has been proven in other literature. However, the proofs in this article are based on new methods. The equality in this article is the proof of the common cause. At the current stage of our society development of the national economy systematically studying the laws and trends in the economy mathematical modeling is a powerful logical tool. Improve the negative consequences of the financial and economic crisis qualification in economics and mathematics experts play an important role. It aims to explore areas of the economy through computer technology, to identify the condition of studied objects, to predict them, the ability to develop and adopt scientifically-based management decisions will give. Thus, it is optimal in the national economy and sectors The major part of decision making is economic mathematical modeling based on the goal of the ongoing global economic reforms Increasing productivity, wise from scarce resources use, innovation in production increasing the competitiveness of products is composed of The global financial and economic crisis is the solution of these priorities and uncertainty arising in the market economy production companies and firms in the conditions of risk The rational use of scarce resources, analysis of the product group by competitors control of behavior of enterprises and firms, production of enterprises maximizing utilization and minimizing costs Optimization of Business Plan and Strategies, Basic Economics performance indicators, inflation and unemployment learning is important. I hope the inequality in this article will be widely used in the economy in the future.

\section{References}

1. Horn RA, Johnson CR (1989) Matrix analysis. Cambridge University Press. 
2. Gantmacher FR (1998) The Theory of Matrices.1.

3. Lo-ken $\mathrm{H}$ Hormonal analysis of the functions of many.

4. Complex variables in classical regions. Moscow Science.

5. Madraximov RM, Atayev FK (2008) Matrix analogue of Koshi-Bunyakovskiy inequality. Khorezm Academy News letters.

6. Dehghan M, Hajarian M (2008) An iterative algorithm for the reflexive solutions of the generalized coupled Sylvester matrix equations and its optimal approximation. Journal of Applied Mathematics and Computation 202: 571-588.

7. Dehghan M, Hajarian M (2008) An iterative algorithm for solving a pair of matrix equations $\mathrm{AYB}=\mathrm{E}, \mathrm{CYD}=\mathrm{F}$ over generalized Centro-symmetric matrices. Journal Computers \& Mathematics with Applications 56: 3246-3260.

8. Wang QW (2005) Bisymmetric and Centro symmetric solutions to systems of real quaternion matrix equations. Computers and Mathematics with Application 49: $641-650$.

9. Wang QW, Zhang F (2008) The reflexive re-nonnegative definite solution to a quaternion matrix equation. Electronic Journal of Linear Algebra 17: 88-101.

10. Wang QW, Zhang HS, Yu SW (2008) On solutions to the quaternion matrix equation $A X B+C Y D=E$. Electronic Journal of Linear Algebra 17: 343-358.

11. Ding F, Liu PX, Ding J (2008) Iterative solutions of the generalized Sylvester matrix equations by using the hierarchical identification principle. Applied Mathematics Computation 197: 41-50.
12. Peng $X Y$, Hu $X Y$, Zhang $L$ (2007) The reflexive and anti-reflexive solutions of the matrix equation $\mathrm{AXBCH}=\mathrm{A}$. Journal Computational Applied Mathematics 200: $749-760$.

13. Ramadan MA, Abdel NMA, Bayoumi AME (2009) On the explicit solution of the Sylvester and the Yakubovich matrix equations. Journal Mathematical and Computer Modeling 50: 1400-1408.

14. Zhou B, Li ZY, Duan GR, Wang Y (2009) Weighted least squares solutions to general coupled Sylvester matrix equations. Journal Computational Applied Mathematics 224: 759-776.

15. Zhou B, Duan GR (2008) On the generalized Sylvester mapping and matrix equations. Systems \& Control Letters 57: 200-208.

16. Ding $F$, Chen $T$ (2005) Gradient based iterative algorithms for solving a class of matrix equations. IEEE Transactions on Automatic Control 50: 1216-1221.

17. Ding F, Chen T (2005) Hierarchical gradient-based identification of multivariable discrete-time systems. Automatica 41: 315-325.

18. Ding F, Chen $T$ (2005) Iterative least squares solutions of coupled Sylvester matrix equations. Systems Control Letter 54: 95-107.

19. Wu AG, Li B, Zhang Y, Duan GR (2011) Finite iterative solutions to coupled Sylvester-conjugate matrix equations. Applied Mathematical Modeling 35: 1065- 1080.

20. Wu AG, Lv L, Hou MZ (2011) Finite iterative algorithms for extended Sylvesterconjugate matrix equation. Mathematical Computational Model 54: 2363-2384. 